\title{
$\mathrm{CiSj}$
}

\section{MINGLING THE CONTEXTUAL INFORMATION IN IMPROVED MULTIDIMENSIONAL RECOMMENDATION SYSTEM}

\author{
Mohammad Husain \\ AZAD Institute of Engineering and Technology Lucknow (UP), India \\ E-mail: mohd.husain90@gmail.com
}

\begin{abstract}
Recommender systems utilize the times of yore experiences and preferences of the target customers as a basis to proffer personalized recommendations for them as well as resolve the information overloading hitch. Personalized recommendation methods are primarily classified into content-based recommendation approach and collaborative filtering recommendation approach. Both recommendation approaches have their own advantages, drawbacks and complementarities. Because conventional recommendation techniques don't consider the contextual information, the real factor why a customer likes a specific product is unable to be understood. Therefore, in reality, it often causes a decrease in the accuracy of the recommendation results and also persuades the recommendation quality. In this paper, we propose the integrated contextual information as the foundation concept of multidimensional recommendation model and use the Online Analytical Processing (OLAP) ability of data warehousing to solve the contradicting tribulations among hierarchy ratings. This work hopes that by establishing additional user profiles and multidimensional analysis to find the key factors affecting user perceptions, it would increase the recommendation quality.
\end{abstract}

Keywords: Multidimensional Recommendation, Recommender System, Contextual information, Collaborative filtering

\section{INTRODUCTION}

Recommender systems use the earlier period experiences and preferences of the target customers as a foundation to provide personalized recommendations for them and to solve the information overloading problem. The recommender system is not only limited to E-commerce. It is also applicable for searching the most appropriate results in various search systems used in libraries these days. Now a days, mainstream of the researches are focused on the improvement of the algorithm of traditional recommender systems but they all have a similar drawback. Majority of the recommender systems use the gathered data under similar environment to provide recommendations. It was discovered, in an actual experience, that if only customer's past behaviors were considered and the contextual information were ignored, it often caused suspicion in the recommendation results [8]. In recent years, there were also numerous studies proving that, to improve a recommender system, it should all begin in data collection and profile establishment. In the inferring process, the upshots of contextual information should also be considered and be used as the norm of recommendation to provide appropriate recommendation results. Therefore, this research uses the multidimensional recommendation model [1] as the foundation to establish a recommendation structure with multidimensional data collection and analysis ability and solve the book recommendation problems with the use of hierarchy processing and aggregate calculating capabilities. The purposes of this research are:

a. To apply the contextual information in the recommender system, establish a MD recommendation model and discuss its capabilities.

b. To establish rating along with calculating algorithm of the MD recommendation model.

c. To use multi-facets in demonstrating how MD recommendation model ratings forecast the results

\section{LITERATURE REVIEW}

Conventional recommender methods consisted of content-based approach, collaborative filtering approach, knowledge-based approach, utility-based approach and hybrid approach [13][14][15]. Under its application in different domains, customers (C) and items (I) were mainly used as the entities of the recommender system. The recommender system would first acquire the ratings of customers toward items they have already experienced to examine 
their interests and preferences then provide recommendations from items of the same classification that haven't been rated by the customers. In other words, traditional recommender system could be shown as the values of twodimensional " $C \times I$ " matrix and it also computed the rating function of all the customers toward the items $r(c, i)$. It can be shown as $R:$ Customers $\times$ Items $\rightarrow$ Ratings. Adomavicius and Tuzhilin [3] defined the computed range of the rating function as the Recommendation Space (RS). Under R: Customers $\times$ Items $\rightarrow$ Ratings, customers and items determine the ratings so customers and items are included in the RS. In the personalization applications of recommender system, the best recommendation combination between customers and items in the past researches already cannot satisfy the actual needs.

To extend the range of the recommendation space, Adomavicius et al. [5] used the multidimensional space in expressing the rating function. This type of recommendation method where many contextual factors were considered was called multidimensional recommendation model (MD model). The multidimensional capabilities indicate the capabilities of storage and operation of multidimensional data and OLAP capabilities in data warehouses. Because OLAP has the capability of hierarchy aggregate calculating in every dimension [9], it could solve the contextual data handling problem of the recommender system. Therefore, Adomavicius and Tuzhilin [2] integrated the multidimensional data handling capability of OLAP into the recommender system and defined three basic characteristics and capabilities of an MD model, namely: (1) multiple dimensions, (2) profiling capabilities, and (3) aggregation capabilities.

\section{RESEARCH METHODOLOGY}

\subsection{MULTIDIMENSIONAL RECOM- MENDATION STRUCTURE}

The proposed multidimensional recommendation structure of this paper is shown in figure 1. The multidimensional recommendation is utilized as a foundation to establish a absolute multidimensional recommendation model.

\section{i. Recommendation Module}

Since the ratings of customers toward an item might belong to different hierarchies, the recommender system uses the multidimensional recommendation foundation to predict the unknown rating. Here we use the concept of content-based recommendation to establish the dimension profile.
Each dimension profile incorporates the attribute properties and its weighted value. Higher weighted value specify the more important the attribute is. In the primary stage, the recommendation module compares the customer profile (preferences and rules) and the weighted value of attribute in the dimension to determine the customer list (CL) with similar preferences. In the subsequent stage, the customer list (CL) produced from stage 1 is used as the subject of comparison in multidimensional collaborative filtering recommendation. Dimensionality reduction [2] is used to handle the ratings of customers. It is also used to analyze the rapport of target customers and similar customer list to locate the target items for the recommendation list (RL). The final stage determines the key dimensions. It uses the multi-facet to exhibit the properties of key dimensions as the explanation of the recommendation result.
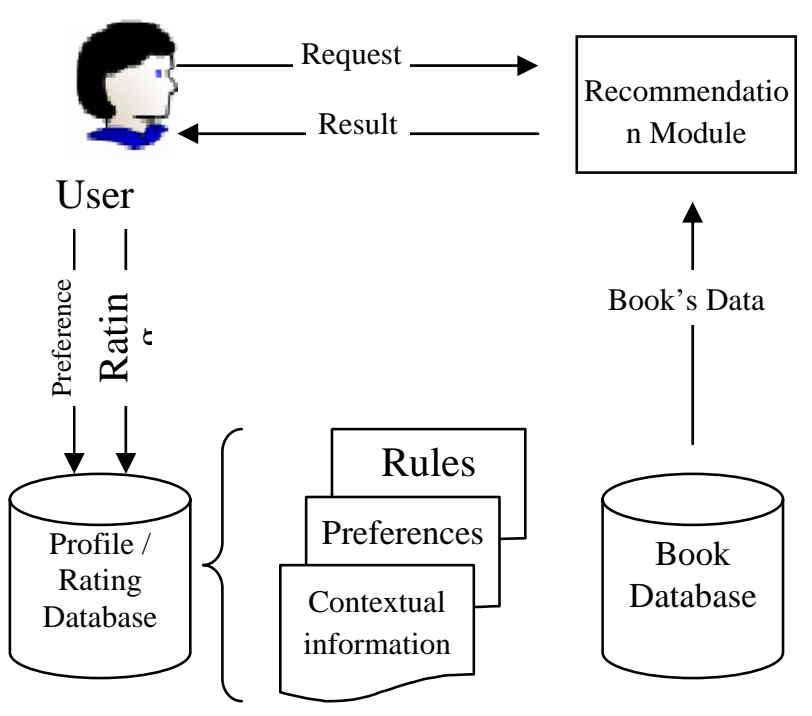

Fig. 1 - Multidimensional Recommendation Structure

ii. Profile database and rating database

The profile database stores the profiles of customers and contextual dimensions (category, stream etc.) together with properties, characteristics and operation methods. The rating database records the ratings of the customer for an item. It is deemed as an important reference of the system to deduce rating.

\section{iii. Book database}

This research establishes a Book recommender system environment that considers five contextual information namely customer, Book, Category and Stream as the multidimensional recommendation foundation. Because the hierarchy ratings have a problem of many rating selections, therefore, this research assumes category and stream as a single value attribute. The book dimension could be 
established to include multi-value or hierarchical attributes. The multi-facet exposed to be able to provide a hierarchical rating selection. The definitions of the dimensions are:

a. Customer: customer is represented by c. Customer properties comprise static and dynamic properties.

b. Book: book is represented by i. It represents the recommendation target as the items indicated in the above which include multiple attributes.

c. Category: category is represented by t. It is description of type like periodicals, journals, news papers, E-Books etc.

d. Stream: It is represented by p. This dimension includes streams like medical, science, political etc.

\subsection{FOUNDATION OF MULTIDIMEN- SIONAL RECOMMENDATION}

This research uses the three characteristics of the multidimensional recommendation environment proposed by Adomavicius [3] as the foundation. The MD model includes the four basic characteristics and capabilities: (1) multiple dimensions, (2) profiling capabilities, (3) aggregation capabilities, and (4) multi-facet capabilities. For the multidimensional recommendation model to have a high-quality recommendation capability, We use the four characteristics and capabilities as the foundation of the multidimensional recommendation system.

\subsection{MULTIPLE DIMENSIONS}

The dimension represents the range of the recommendation space (RS). The conventional recommendation method considered only two dimensions specifically customer and item. The concept of multi-dimension is extended from the data warehouse and OLAP using multi-facet to view the data. If the rating computation would be used to handle the recommendation problem, traditional two-dimensional recommender system can be represented as $R$ : Customers $\times$ Items $\rightarrow$ Ratings. It means that the rating was determined by customer and item. The $r(c, i)$ values of two-dimensional " $C \times I$ " matrix was the inferring purpose of the recommendation and used the dimensions customer and items as its foundation. This work adds the other contextual information and each context are represented by one dimension. The recommendation space (RS) is shown as $R S=D_{1} \times D_{2} \times \ldots \times D_{n}$. Every dimension has its individual format, attributes and operation method. Its attributes are utilized as the major description of the dimension. It is expressed as $D_{i} \subseteq A_{i 1} \times A_{i 2} \times \ldots \times A_{i j} . \quad A_{i j} \quad$ represents the properties included in the dimension $D_{i}$. Additionally, the attributes could also be a set.

The dimension and attributes comprise the concept of hierarchy. In the dimensions books and category, the hierarchy of the book is based on the book classification structure (i.e. product classification). The representation method of the two-dimensional rating function is utilized as the foundation. The multidimensional recommendation model rating could be shown as $r\left(D_{l}, D_{2}, \ldots, D_{n}\right)$. The influencing key dimensions $\left(\mathrm{D}_{1}, \mathrm{D}_{2}, \ldots\right)$ are used to determine the value of the recommendation function. Customers and items are the two basic dimensions that are indispensable in a multidimensional recommendation; therefore, the definition represented by the rating function is the level of likeness of a customer towards an item in a context. For example, if a recommendation space includes the three dimensions customer, book and category, its rating function is shown as $r(c, i, t)$. From r(Husain, Software Project Management System, EBook) $=7$, it is shown that Husain likes to read books on computers. It expresses the customer's preference and explains the customer behavior.

In recommendation space, the key dimensions could be used as ratings just as if the purpose of the traditional recommender system lies within $C \times I$ matrix, the rating function $\left(r\left(D_{1}, D_{2}, \ldots, D_{n}\right)\right)$ of the MD model lies within a multidimensional matrix. For example, if the recommendation space includes customer, book and category, the rating $r(c, i, t)$ lies in a three-dimensional space $C \times I \times T$. The threedimensional recommendation space is given as follows:

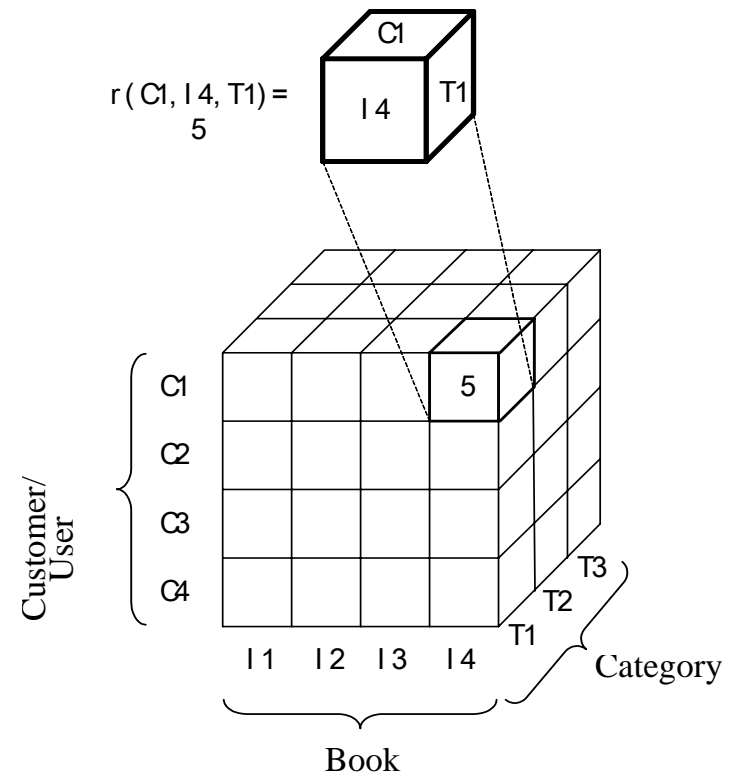

Fig. $2-\mathrm{C} \times \mathbf{I} \times 1$ recommendation space 
The hitch of multidimensional recommendation is similar with that of the traditional recommendation which is to calculate the rating function in the matrix. The first difficulty it encountered is choice of dimensions. The recommendation space may comprise multiple dimensions but not all dimensions are applicable. In other terminology, not all the contextual information is influential. To select the key dimension that is most significant to the recommendation result is a really valuable topic for research. We use category and stream as the key dimensions. After analyzing the contextual information, many additional interesting tribulations are discovered. Aside from explaining the tribulations from may different angles, analysis operations could be used to make appropriate recommendations.

\subsection{PROFILING CAPABILITIES}

We apply the concept of profile construction in the multidimensional recommendation model here. Traditional profile construction techniques are mostly applied in describing the characteristics of customers and items. When applied in the multidimensional recommendation environment, there are also changes in the establishing capabilities and patterns of the profiles. The technique for establishing the multidimensional profile is more complicated and considers more problems than the traditional one. According to its capabilities of the multidimensional profiling techniques shown above, we believe that it would surely provide better recommendation results. The multidimensional recommendation model applies more appropriate dimensional profiles as recommendation foundation. One of its advantage is it can provide appropriate and accurate recommendation results. Considering contextual information not only helps increase the accuracy of the recommendation result, it is even better for establishing rules to explain the preferences of customers with special or unique demands. In addition, another advantage is that the results by the profiling can secure and reduce the recommendation range.

\subsection{AGGREGATION CAPABILITIES}

Aggregation is the main purpose of establishing data cubes in data warehousing. Aggregation is a tool of the traditional analysis. When the dimensions of the data cube increase, it would affect the time of inquiry. By using aggregation, it would focus its analysis on the main information. The hierarchical structure and the aggregation capabilities are combined for the MD model to have similar storing capability like data warehouse, OLAP data analysis and aggregation capabilities. Using book recommendation as the example, one-on-one rating was utilized in the past. Using the hierarchy concept and aggregation, R(Husain,Software Project Management $)=6$ was also similar to the results $r$ (Husain, E-Books $)=6$ or $r$ (male, Software Project Management $)=6$. The ratings of Husain towards EBooks could be utilized to estimate his level of likeness towards E-Books or utilize the ratings of the male population who already read the book "Software Project Management" to estimate r(male, Software Project Management). The mathematical equation would be:

$r($ Husain, E-Books $)=A G G R_{\text {B.type }=e-\text { book }} r($ Husain, B $)$, $B=\left\{\right.$ book $_{1}, \ldots$ book $\left._{n}\right\}$

If Average (AVG) represents the aggregation function (Aggr), formula (1) would be:

$r($ Husain,$E-$ Books $)=A V G_{\text {B.type }=\text {-book }}$ (Husain, B), $B=\left\{\right.$ book $_{1}, \ldots$ book $\left._{n}\right\}$

\subsection{MULTI-FACET CAPABILITIES}

Past recommender systems provided recommendation list according to the forecasted rating levels. It cannot effectively explain the recommendation result and also cannot explain how the recommendation was produced. Especially in handling searching results, Differences of Semantics were utilized to classify searching results and multifacet demonstration was utilized to show searching results in order to conform the needs of users. If only a fixed attribute is utilized for aggregate computation in handling hierarchy ratings, contradicting results would often appear. We believe that the result would not be the fault of aggregation but due to the selection of wrong attribute (type of book) would lead to the wrong result of aggregation. Books involve many properties or attributes. Each attribute could become the reason of selection by the customers, therefore, the numerous attributes of the books should be considered. The aggregation result shown in figure 3 discovers that the attributes "auther" and "year" are similar with the aggregation result. Both sides could provide an appropriate classification and explanation toward the recommendation result. 


\section{could be divided into four parts, shown in figure 4 .}

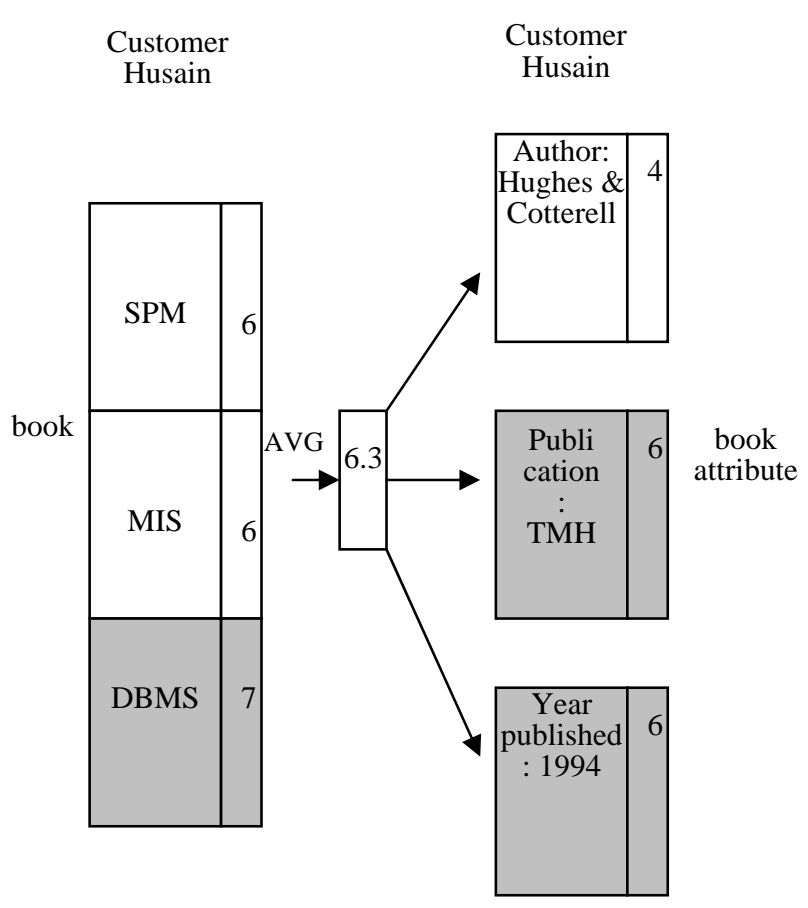

Fig. 3 - Multi-facet aggregation

\section{EXPERIMENT DESIGN}

\subsection{STRUCTURE OF EXPERIMENT SYSTEM AND EXPLANATION}

The proposed multidimensional recommendation is applied in the book recommendation of online library. The subjects of this work are the members of the online library community. Even though most of book information, websites have detailed information of the book, ratings, discussions, etc., it still doesn't have the capability of collecting related contextual information. Therefore, this experiment couldn't directly utilize the rating information of the book information websites for analysis. Due to this problem, we design an experimental website and collect the necessary information to be utilized in this experiment. To verify the feasibility of its structure, we make an experimental book recommendation system to collect the rating of the users toward the book and provide book recommendation according to the recorded rating. Here we focus on the capability of the multidimensional recommendation model and not in the learning mechanism towards improving the outcome of the recommendation result, the capability of the learning mechanism is not added in the experiment process. The experimental system consists of two main capabilities namely book rating and book recommendation. The system structure

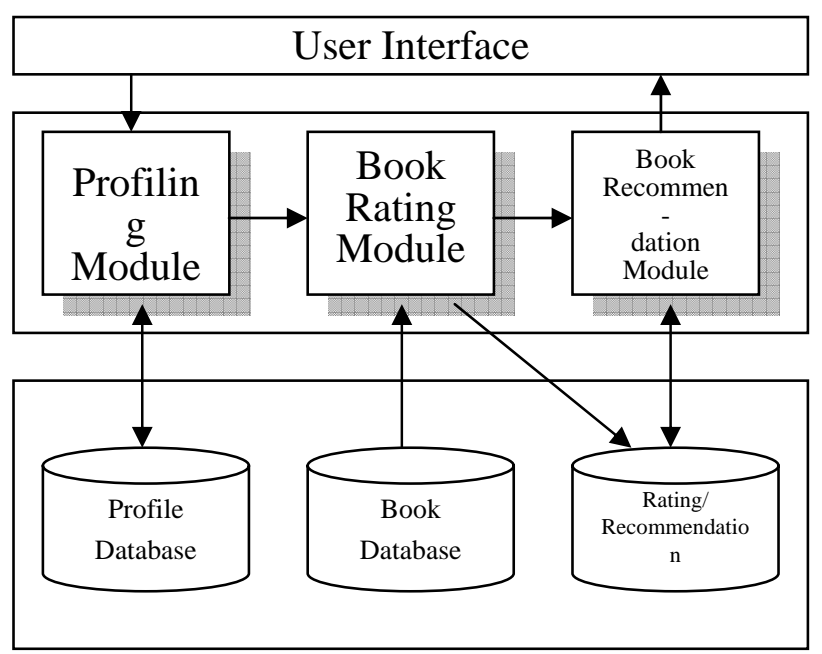

Fig. 4 - Structure of experiment system

Detailed explanations of the parts are explained below:

(1) Database: stores profile information of the users, book information, author and publisher information, rating information, recommendation information and statistics information.

(2) Profiling module of users: includes the three profiles namely book, category and stream. The user should establish his/her personal profile at the beginning of the experiment.

(3) Book rating module: We utilize the collection of digital library of Azad Institute of Engineering and Technology from January 2007 to February 2009 (a total of 309 book' information) as the experiment foundation to provide picture and text book information for users to rate the books. Points 1-7 is utilized to rate the user's interest in the book where 5 and above representing the user is interested in that book. It also records the user's appreciation towards the contextual information of the book.

(4) Book recommendation module: The Book recommendation module is divided into two parts. The first part considers the multidimensional recommendation model of the contextual information. Firstly, it analyzes a possible book list for recommendation according to the profile of the target customer/user. The books in the book list that could be recommended are declared as target books. Users with much variation are eliminated according to the profiles of the target users and search for target users that might be in similar customer list of common rating behavior. The similarity of the target user and possible similar 
customer group are computed. If the similarity value is 0.5 greater than the threshold value, include it in similar customer group. List similar customer list according to the rating of the target book one by one. If similar rating of a target book exceeds three persons, then the forecasted rating of the book can be calculated. Provide recommendation result explanation after comparing the forecasted rating and the aggregated rating of the book attribute. Lastly, list the top five recommended books. The next part is the traditional collaborative filtering recommendation module. The effect of the contextual information is eliminated and just considers the dimensions, user and book. List the top five recommended books.

\subsection{SETTING OF SYSTEM \\ PARAMETERS}

Before continuing with the experiment, first provide a clear setting of the multidimensional recommendation algorithm and the related parameters used in the experiment operation. Table 1 shows the system parameter setting and their description.

\subsection{PROCESS OF THE EXPERIMENT}

The experiment process is shown below -

(1) The first stage of the experiment uses the collected customer ratings as the main purpose of the recommendation computation foundation of stage two. The members of a book discussion area are utilized as the experiment sample. The system in stage one doesn't recommend any books. A total of 40 persons are examined and 1452 book rating information is collected.

(2) The number of examined sample in stage two is 25 and they are different from the 40 samples selected in stage one. The customers are set to rate at least 25 books before proceeding to the other parts of the experiment. The system produces two groups of recommendation results. A total of 10 books are divided equally into 5 multidimensional recommendation results and 5 collaborative filtering recommendation results. The customers separately rate their satisfaction according to the recommendation result and recommendation description The satisfaction level of the recommendation result is divided into 5 points and they could select whether they have watched the book or not. The recommendation description is divided into 7 points.
Table 1. Table of system parameter setting

\begin{tabular}{|c|c|}
\hline Parameter Setting & Description \\
\hline $\begin{array}{l}\text { Number of experiment } \\
\text { sample in stage 1: } 40 \\
\text { persons }\end{array}$ & $\begin{array}{l}\text { Collect book rating } \\
\text { information }\end{array}$ \\
\hline $\begin{array}{l}\text { Number of experiment } \\
\text { sample in stage 2: } 25 \\
\text { persons }\end{array}$ & $\begin{array}{l}\text { Ask } 25 \text { persons to rate the } \\
\text { books, render them to the } \\
\text { multidimensional book } \\
\text { recommendation result, } \\
\text { provide the ratings and } \\
\text { select its level of } \\
\text { satisfaction }\end{array}$ \\
\hline $\begin{array}{l}\text { Threshold value of the } \\
\text { book rating in stage } 2 \text { : } \\
25 \text { books }\end{array}$ & $\begin{array}{l}\text { Set the lowest allowable } \\
\text { value for book rating of a } \\
\text { user. Ensure that the } \\
\text { common rating is greater } \\
\text { than the threshold value }\end{array}$ \\
\hline $\begin{array}{l}\text { Threshold value of the } \\
\text { liked book: } 5\end{array}$ & $\begin{array}{l}\text { Points } 1-7 \text { is used to rate } \\
\text { the user's interest in the } \\
\text { book where } 5 \text { and above } \\
\text { representing the user is } \\
\text { interested in that book }\end{array}$ \\
\hline $\begin{array}{l}\text { Common rating of the } \\
\text { books: } \\
6 \text { books and above }\end{array}$ & $\begin{array}{l}\text { The target user } c \text { and the } \\
\text { possible similar user } c \\
\text { should have } 6 \text { or more } \\
\text { books with common } \\
\text { ratings to be able to } \\
\text { compute for the similarity } \\
\text { value }\end{array}$ \\
\hline $\begin{array}{l}\text { Threshold value of the } \\
\text { similar customers: } 3 \\
\text { and above }\end{array}$ & $\begin{array}{l}\text { To be able to compute for } \\
\text { the forecasted rating, the } \\
\text { number of similar target } \\
\text { customers should be } \\
\text { greater than } 3\end{array}$ \\
\hline $\begin{array}{l}\text { Similarity's threshold } \\
\text { value: } \\
0.5 \text { and above }\end{array}$ & $\begin{array}{l}\text { To compute for the } \\
\text { similarities between the } \\
\text { target customer } c \text { and } \\
\text { possible similar customers } \\
c \text { ', greater than } 0.5 \text { could } \\
\text { already determine the } \\
\text { similar customers }\end{array}$ \\
\hline
\end{tabular}

\section{CONCLUSION}

This research proposes a multidimensional recommendation environment to integrate the contextual information. It also defines the attributes and capabilities of the multidimensional recommendation structure specifically multiple dimensions, profiling capabilities, aggregation capabilities and multi-facet capabilities. With the above said capabilities, the recommender system could simultaneously possess the advantages of content-based recommendation, knowledge-based recommendation, collaborative filtering recommendation and OLAP in segmenting the 
information. Following the improvement of the recommendation structure, it doesn't have to limit its analysis on the customer and product to compute for the recommendation result and it could also handle and determine more complex contextual information as recommendation computation foundation. It could develop improved results if applied in different provinces.

Aside from proposing theories, this work designs a book recommender prototype system to enhance the confirmation of the multidimensional recommendation result. The research results confirm that adopting contextual multidimensional recommendation method could promote the accuracy of the recommendation results. The forecasted ratings and the real customer satisfactory level are pretty close meaning that the proposed recommendation method is good. Besides, most of the viewing behaviors of the users are consistent with the system recommendation results which show that the recommendation result conform with the customers' behaviors. In a short period of time, this study could control the preferences and interests of the customers to produce good recommendation results.

The purpose of this work is to propose a complete multidimensional recommendation structure and process and proposes to solve the contradicting problem of hierarchy rating using multi-facet capabilities. Although the experiment didn't focus on testing and measuring feedback and learning module, we believe that if the module is added in the recommender system and with continuous collection of user rating information, the system could re-compute for the similarity of users and correct the user preferences then re-compute the recommendation result. So, innovative recommendation information could be achieved after a short period of time. Because the system combines multi-facet attributes, aside from producing recommendation results according to the special preferences of the customers, result classification could also be done according to the reason of recommendation and it can even provide recommendation by immediately combining the contextual information

\section{REFERENCES}

[1] Adomavicius G., Sankaranarayanan R., Sen S. and Tuzhilin A., Incorporating contextual information in recommender systems using a multidimensional approach, ACM Transactions on Information Systems, 23 (1) (2005). pp. 103-145.

[2] Adomavicius G. and Tuzhilin A., Toward the next generation of recommender systems: a survey of the state-of-the-art and possible extensions, IEEE Transactions on Knowledge and Data Engineering, 17 (6) (2005). pp. 734-749.

[3] Adomavicius G. and Tuzhilin A., Extending recommender systems: A multidimensional approach, Proceedings of the International Joint Conference on Artificial Intelligence (IJCAI-01), Workshop on Intelligent Techniques for Web Personalization, Seattle, (2001).

[4] Billsus D. and Pazzani M., User modeling for adaptive news access, User Modeling and User-Adapted Interaction, 10 (2-3) (2000). pp. 147-180.

[5] Burke R., Hybrid recommender systems: survey and experiments, User Modeling and User-Adapted Interaction, 12 (4) (2002). pp. 331-370.

[6] Deshpande M. and Karypis G., Item-based Top-N recommendation algorithms, $A C M$ Transactions on Information Systems, 22 (1) (2004). - pp. 143-177.

[7] Herlocker J. L., Konstan J., Terveen L. and Riedl J., Evaluating collaborative filtering recommender systems, ACM Transactions on Information Systems, 22 (1) (2004). - pp. 5-53.

[8] Jung S., Harris K., Webster J. and Herlocker J. L., SERF: integrating human recommendations with search, Proceedings of the 13th Conference on Information and Knowledge Management, (2004). - pp.571580.

[9] Kimball R., The Data Warehouse Toolkit, John Wiley \& Sons, Inc., 1996.

[10] Li Y., LuL. and Xuefeng L., A hybrid collaborative filtering method for multipleinterests and multiple-content recommendation in e-commerce, Expert Systems with Applications, 28 (1) (2005). - pp. 67-77.

[11] Linden G., Smith B. and York J. Amazon.com recommendations item to item collaborative filtering, IEEE Internet Computing, 7 (1) (2003). - pp. 76-80.

[12] Shih Y. Y. and Liu D. R., Hybrid recommendation approaches: collaborative filtering via valuable content information, Proceedings of 38th Hawaii International Conference on System Sciences, Big Island, (2005).

[13] Tang T. Y., Winoto P., Chan K. C., On the temporal analysis for improved hybrid recommendations, Proceedings of the IEEE/WIC (WI'03), Halifax, (2003). - pp. 214220.

[14] Tiraweerakhajohn C. and Pinngern O., Finding item neighbors in item-based collaborative 
filtering by adding item content, Proceedings of the 8th International Conference on Control, Automation, Robotics and Vision, Kunming, (2004). - pp. 1674-1678.

[15] Yang W., Wang Z. and You M., An improved collaborative filtering method for recommendations generation, proceedings of the 2004 ieee international conference on systems, man and cybernetics, The Hague, 5 (2004). - pp. 4135-4139.

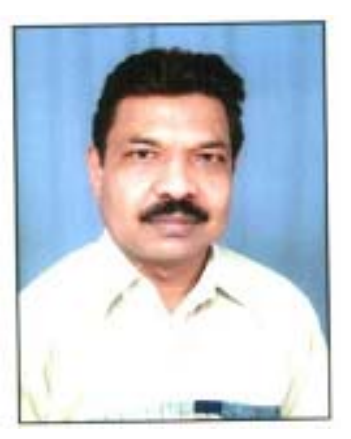

Dr. Mohd. Husain: He is working as Professor at $A Z A D$ Institute of Engineering and Technology, Lucknow, India. He got his Master's Degree from UP Technical University \& Ph.D. Degree work from Integral University in 2008. He has more than 15 years teaching experience and 10 years research experience in the field of Data mining. He has published more than 100 International and National publications. 